\title{
Hazard Analysis and Error Proofing in Spacecraft General Assembly
}

\author{
Botao $\mathrm{Xu}^{1, *}$, Zhikai Fang ${ }^{1}$, Yugang $\mathrm{Liu}^{2}$, Tao Guo ${ }^{3}$, Zhengyi Xiao ${ }^{3}$, Bin Zhang ${ }^{3}$ and Weibing Dai $^{4}$ \\ ${ }^{1}$ Beijing Institute of Spacecraft Environment Engineering, Beijing, 100094, China \\ ${ }^{2,3}$ Beijing Engineering Research Center of the Intelligent Assembly Technology and Equipment for Aerospace Product, Beijing \\ ${ }^{4}$ Beijing Institute of Spacecraft Environment Engineering, Beijing, 100094, China \\ *Corresponding author
}

\begin{abstract}
The paper analyzes occurrence of hazardous factors in spacecraft general assembly process, and analyzes the level and effect of hazards in various general assembly units. The methodology of designing error proofing for general assembly is discussed, a database of error control elements is established. Process error analysis and error proofing technological evaluation is studied to ensure the quality of spacecraft general assembly.
\end{abstract}

Keywords-spacecraft general assembly; hazard analysis; error proofing

\section{INTRODUCTION}

In recent years, although the extremely serious quality accidents resulting in the crash of spacecraft and the loss of human life rarely happened during the spacecraft assembly process, the slight injuries of personnel and the damages of products which can be repaired or acceptable frequently occurred, such as the bump of the engine and the sparking of storage battery in the spacecraft assembly process. With the continuous development of the space technology in our country, the application of new technology and materials, as well as the research task quantities of the spacecraft increase dramatically, and the occurrence probability of quality problems during the spacecraft assembly process is also on the rise [1]. How to ensure the quality safety (QS) of the spacecraft assembly using the scientific quality control theory based on the acquaintance of the accident features is a new subject faced by the major of spacecraft assembly in the future stable development.

The error proofing of spacecraft assembly refers to the ability to avoid the occurrence of unforeseen incidents which can cause the life loss of personnel, or the damages of spacecraft products, process equipment and plant facilities. Thereinto, all the unforeseen incidents are collectively called accidents. The causes of the accidents are generally the quality problems, and the risk refers to the possibility of an accident represented by the error-prone probability and the seriousness of the quality problem [2].

The work of error control emphasizes on the prevention. Therefore, fully distinguishing the faults factors in the assembly process, reinforcing the technical research and the quality control measures of spacecraft assembly will be of great significance to ensure the quality safety of the spacecraft assembly.

\section{The Risk Research IN THE Assembly PROCESS}

\section{A. The Analysis of Faults Factors}

The main tasks of the spacecraft assembly include thermal control implementation, pipe welding, cablecasting, equipment installation, grounding implementation, large-scale hoisting, spacecraft transfer, high-altitude work, high pressure work, precision measurement, leak test, qualitative characteristic measurement and so on. In order to control the risk of spacecraft assembly, the faults factors of spacecraft assembly are preliminarily analyzed using preliminary hazard analysis (PHA) to distinguish the potential faults points in the production systems and determine their hazard ratings. In general terms, the faults factors of the whole assembly process include the personnel, machinery, material, method, environment and so on. Every factor can also be hierarchically divided into many secondary influencing factors, thus the whole assembly process can be decomposed into the summation of many factors according to the stipulated granularity. A relatively complete and clear faults factors analysis graph can be obtained by determining and screening these factors according to the classification rules of risk and hazard, screening out the risk factors and adding them into the general drawing of factors (see Figure 1).

\section{B. Identification of Faults}

According to Figure 1, it can be clearly seen that the faults factors in the spacecraft assembly process are mainly derived from the following three aspects: 1) personnel factors: namely the factors that will affect the normal work because the operation personnel cannot adapt to the requirements of their jobs due to the physiological factors, psychological factors, and capability factors; 2) objective factors: including the defects and fallibility existing in the power source of the product itself, the mixed factors of the intrinsic defects and the usage of the tooling equipment and tools; 3) environmental factors: namely the effects of the assembly conditions such as temperature, humidity, illumination, noise and work space on the operation personnel. 


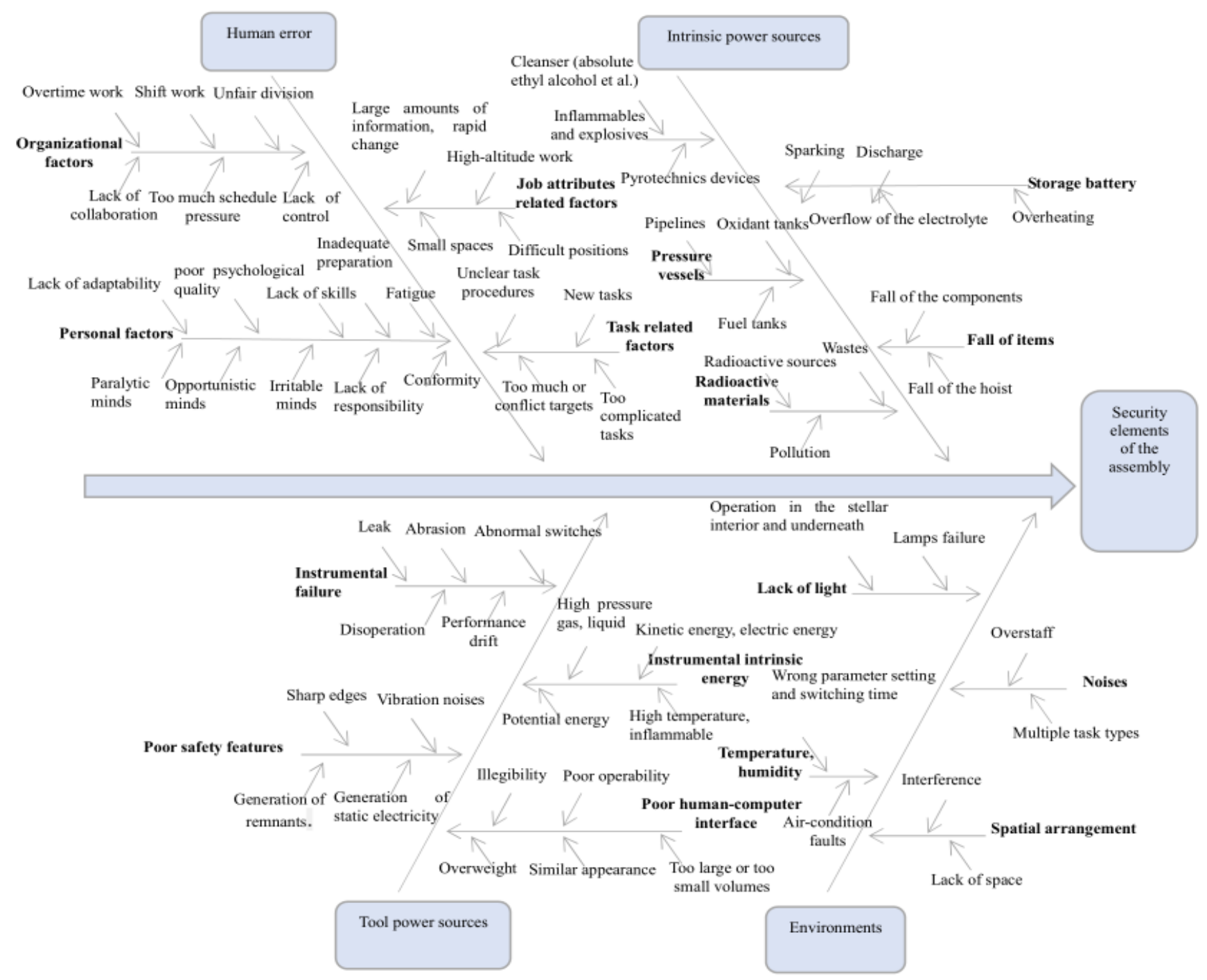

FIGURE I. SPACECRAFT AIT FAULTS FACTOR ANALYSIS: STRUCTURAL GRAPH

After analysis, the faults factors in the assembly process mainly include: personnel (incorrect operation), superfluous objectives (generation), static electricity (hazard), initiating explosive devices and chemicals (flammable and explosive), high-altitude work (fall), high pressure work (explosive), hoisting work (fall of objects), radioactive source (radiation and pollution in installation process), electromagnetic radiation.

According to the statistics and analysis of the quality issues and accidents of the spacecraft assembly in our country, among the faults factors in the spacecraft assembly process, the factors that cause the slight injury of the personnel while their jobs are not affected or the existing hidden dangers account for $40 \% \sim 50 \%$, and the explosions (incorrect operation of the initiating explosive devices, incorrect operation of the high pressure work) that cause loss of life and damage of products account for 1\%. In Table 1, 5 grades of the assembly working units have been divided based on the faults degree: catastrophic, severe, medium, mild and slight, and the faults factors as well as the possible hazard to the personnel and products have also been analyzed. 
TABLE I. FACTORS OF FAULTS LISTED BY AIT CELL

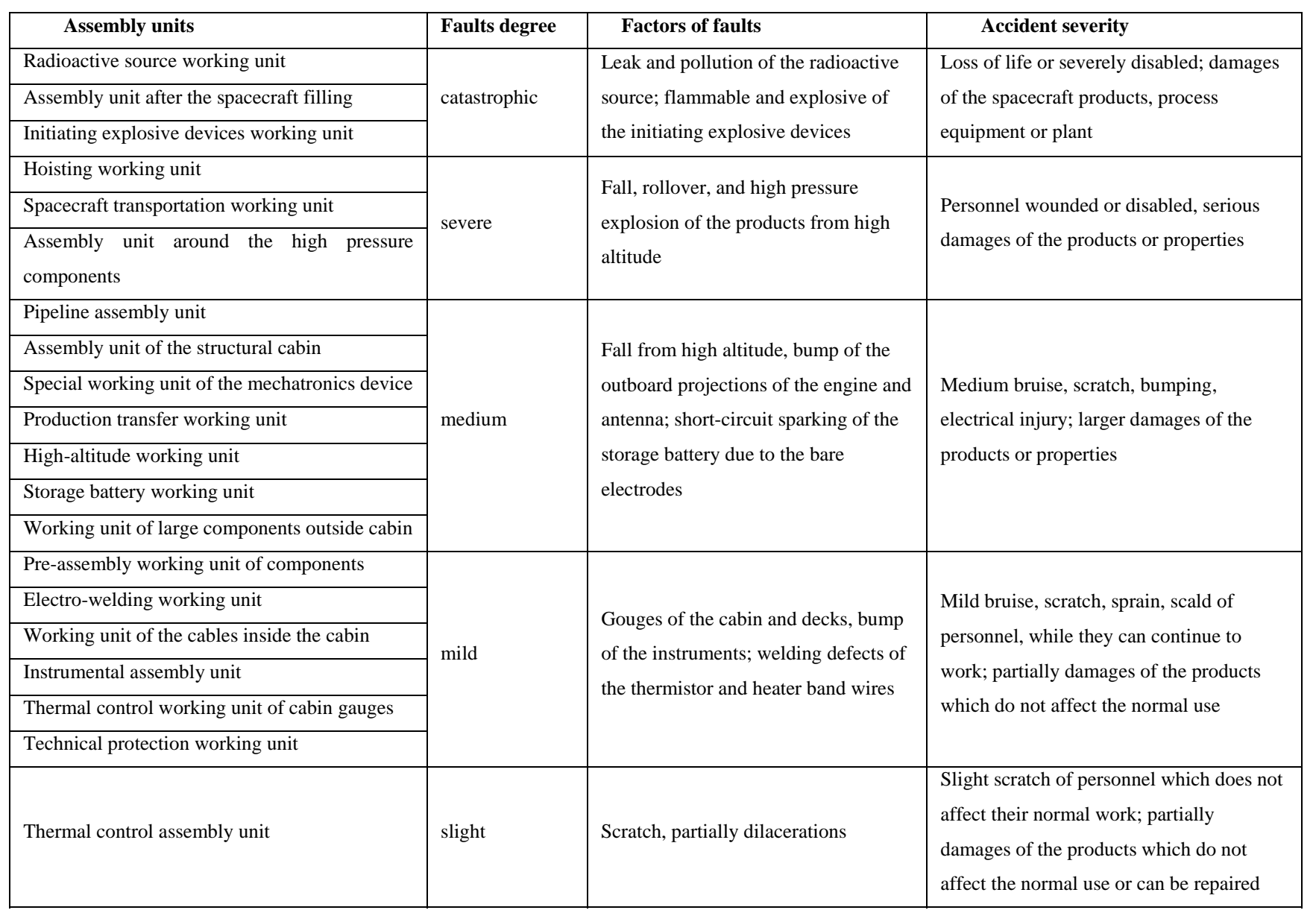

\section{The ERror ProOfing RESEARCH OF THE SPACECRAFT}

According to the analysis of the previous assembly accidents, it can be found that the current error proofing measures of the assembly process are inadequate, and the main problems are:

\section{A. Analysis of Process Deficiency}

In the course of several decades of development of the spacecraft assembly, the typical thought of people is that any problems occurred in the assembly production process take the damage degree of the spacecraft products as the key consideration, and the analysis of "faults problems" belongs to the "quality issues". As a result, in the error proofing design of the assembly process, the technologists are mainly concerned on the quality control points. In the assembly technological procedure, there are quality control requirements in every process, while the error control elements merely run through the operation process contents in the form of notes and precautions, leading to the weak guidance of the assembly error proofing and control, which is difficult to make the advanced prevention, control and warning work.

\section{B. Improvise}

The error proofing knowledge and the experience level of the assembly technologists are different, and the lack of error proofing theory is ubiquitous. The ability of pre-analysis of the potential errors is not strong and comprehensive. Moreover, the communication among different technologists in various models is inadequate, resulting in the different error proofing guidance levels in the current assembly process, which is still a gap between the requirements of comprehensive control of risk.

We should make the comprehensive, intensive and systematical technical analysis on the faults points in the assembly process using the error proofing evaluation theory to improve the control capability of the faults factors, on the premise of "people-oriented" error control concept [3]. Formalizing the error proofing factors of the assembly process is the fundamental object of enhancing the assembly production technical support.

\section{Research Approach}

In the error proofing research of the assembly process, the existing error proofing related standard specification and documents are firstly combed, and the error proofing factors in the spacecraft assembly process are studied and analyzed. After 
that, the analytical and statistical work are performed on the relevant content of the technological procedure to systematically distinguish the probable abnormal working conditions (hazard) occurred in terms of the technological process, operation objects, and operation personnel. The relevant faults points at all levels are found out, and the necessary measures are taken to eliminate and reduce the occurrence of the abnormal working conditions (hazard), consequently making the spacecraft assembly process under control [4]. Finally, the error proofing factors controlling database of the assembly technological procedure is established to organically combine the error proofing factors and the technological procedure.

\section{Establishment of the Control Elements Database}

The error proofing factors of the spacecraft assembly operation process are studied, and particular stress should be laid on the study of the error proofing factors of the typical assembly units and the corresponding four-dimensional error proofing factors of "operation personnel, tooling facilities, operation environments, faults management”. The grading standards of the fallibility in the spacecraft assembly process should be established. According to the specific assembly practical experience and error control characteristics of different spacecraft models, the standardized, formalized and systematical management should be carried out on the previous process reports of the assembly technological procedure error control elements which are distributed across various processes of the different spacecraft assembly technological procedures using a unified database management method, and the "The control strategy table and database of the standardized reports of the error proofing factors of the spacecraft assembly technological procedures" should be formulated and established to further promote the formalization degrees of the error control elements in the assembly process of the spacecraft assembly technological procedures.

\section{E. The Reflection of Preventive Measures in the Technological Procedure}

The format template of "error proofing control card" of the assembly technological procedures should be worked out to provide the assembly technologists for the relatively normative alternative template sets of the error proofing elements. The error proof measures should be reflected in the technological procedures to achieve the guiding role of the technological procedures on the site operations.

\section{CONCLUSIONS}

In this paper, the faults factors existing in the production systems of the spacecraft assembly are distinguished, and the realization approaches of the error proofing study on the spacecraft assembly processes are proposed, according to the "prevention first" error proof basic principle and the "people oriented" error proofing concept. The unified specification is suggested, and it should be implemented throughout the error proofing standard systems of the assembly and the assembly technological procedures as part of the technological error proofing designs, thereby providing guarantees for the quality of spacecraft assembly.

\section{REFERENCES}

[1] Lawrence P. Chao, Kosuke Ishii. Design Process Error Proofing: Failure Modes and Effects Analysis of the Design Process [J], Journal of mechanical design, 2007, Vol 129(5).

[2] Safarpour, S.; Veneris, A.; CSMDL: Abstraction and Refinement Techniques in Automated Design Debugging [C]. Design, Automation \& Test in Europe Conference \& Exhibition (DATE 2007), 2007; Nice, France

[3] Zhizhong Yin. A complex-error and phase-error constrained leastsquares design of FIR filters with reduced group delay error [C]. Chinese Control and Decision Conference (CCDC 2009).[Vol.1] 